\title{
ESTUDIO DE LA VARIACIÓN EPIGENÉTICA CRANEOFACIAL EN EL HUMEDAL DEL PARANÁ INFERIOR (ARGENTINA) DURANTE EL HOLOCENO TARDÍO
}

\author{
A STUDY OF THE CRANIAL AND FACIAL EPIGENETIC VARIATION IN THE \\ LOWER PARANÁ WETLAND (ARGENTINA) DURING THE LATE HOLOCENE
}

\author{
Bárbara Mazza1 y Mariana Fabra²
}

\begin{abstract}
Los estudios sobre la presencia de límites sociales o de diferentes sociedades y poblaciones en el humedal del Paraná inferior (Argentina) fueron siempre inferidos a partir de la base material del registro arqueológico, ya sea por diferencias estilísticas o bien por la presencia/ausencia de ciertas características arqueológicas materiales. El presente trabajo tiene como objetivo complementar dichos estudios mediante el análisis de rasgos epigenéticos craneofaciales. Para esto se analizaron 39 individuos provenientes, por un lado, del norte y sur del Paraná Guazú y, por el otro, de los subsectores ambientales denominados Planicies inundables y Delta inferior. Los resultados obtenidos muestran diferencias estadísticamente significativas entre estas dos últimas unidades ambientales, dadas por la presencia de la foramina infraorbital múltiple y del hueso wormiano epiptérico entre los individuos de Planicies inundables y su completa ausencia entre aquellos del Delta inferior. Estos resultados, si bien preliminares, son un gran avance para la arqueología regional, ya que es la primera vez que la existencia de diferencias biológicas puede ser interpretada como la coexistencia de diferentes sociedades a través de análisis que exceden la comparación intrarregional de la variabilidad artefactual.
\end{abstract}

Palabras claves: humedal del Paraná inferior, variabilidad biológica, rasgos epigenéticos craneales, límites sociales.

Studies on the presence of social boundaries or of different societies and populations in lower Paraná wetlands (Argentina) have always been inferred from the material basis of the archaeological record, either from stylistic differences or by the presence I absence of certain archaeological features. This paper aims to complement these studies through the analysis of epigenetic craniofacial traits. Thirty nine individuals, from the north and south of the Paraná Guazú River and, from the Flood plains and lower Delta environmental subsectors, were analyzed. The results show statistically significant differences between the latter two environmental units, given by the presence of multiple infraorbital foramina and epipteric bone among individuals from the Flood plains, and their complete absence among those from the lower Delta. These results, although preliminary, are a breakthrough for the regional archaeology, as it is the first time the existence of biological differences can be interpreted as the coexistence of different societies through analyses that exceed the intraregional comparison of artifactual variability.

Key words: Lower Paraná wetland, biological variability, epigenetic traits, social boundaries.

El humedal del Paraná inferior (HPI) se encuentra dentro de la región geográfica del noreste argentino, específicamente al sur de la provincia de Entre Ríos y noreste de la provincia de Buenos Aires $\left(33,7^{\circ}-34,4^{\circ}\right.$ latitud sur y $58,3^{\circ}$ $59,6^{\circ}$ longitud oeste). Las crónicas de los siglos XVI y XVII señalan que esta área estuvo habitada por varias sociedades cazadoras-recolectoras, cada una de ellas con un nombre propio, y horticultoras, comúnmente conocidas como Guaraníes (Lopes de Sousa 1927; Lozano 1874; Oviedo y Valdés 1944; Schmidl 1948). Dichas sociedades son descritas a partir de un conjunto de rasgos culturales que eran compartidos por toda la región, como así también de ciertos elementos de su cultura material o costumbres que los diferenciaban (Tabla 1).

Esta información histórica puede profundizarse temporalmente mediante el registro arqueológico, el cual señala que hacia los 2.300 años ${ }^{14} \mathrm{C}$ a.p. se encontraban asentadas sociedades con una subsistencia basada principalmente en la caza, pesca y recolección (Loponte et al. 2012). Estudios arqueológicos recientes demostraron la presencia de diferencias intra e interregionales respecto de la

\footnotetext{
1 Instituto Nacional de Antropología y Pensamiento Latinoamericano, CONICET. 3 de febrero 1378 - Buenos Aires, Argentina. barbara_mazza@yahoo.com.ar

2 Instituto de Antropología de Córdoba/CONICET, Museo de Antropología (FFyH, UNC). Av. Hipólito Yrigoyen 174, CP.5000, Córdoba, Argentina. marianafabra@gmail.com
} 
692

Bárbara Mazza y Mariana Fabra

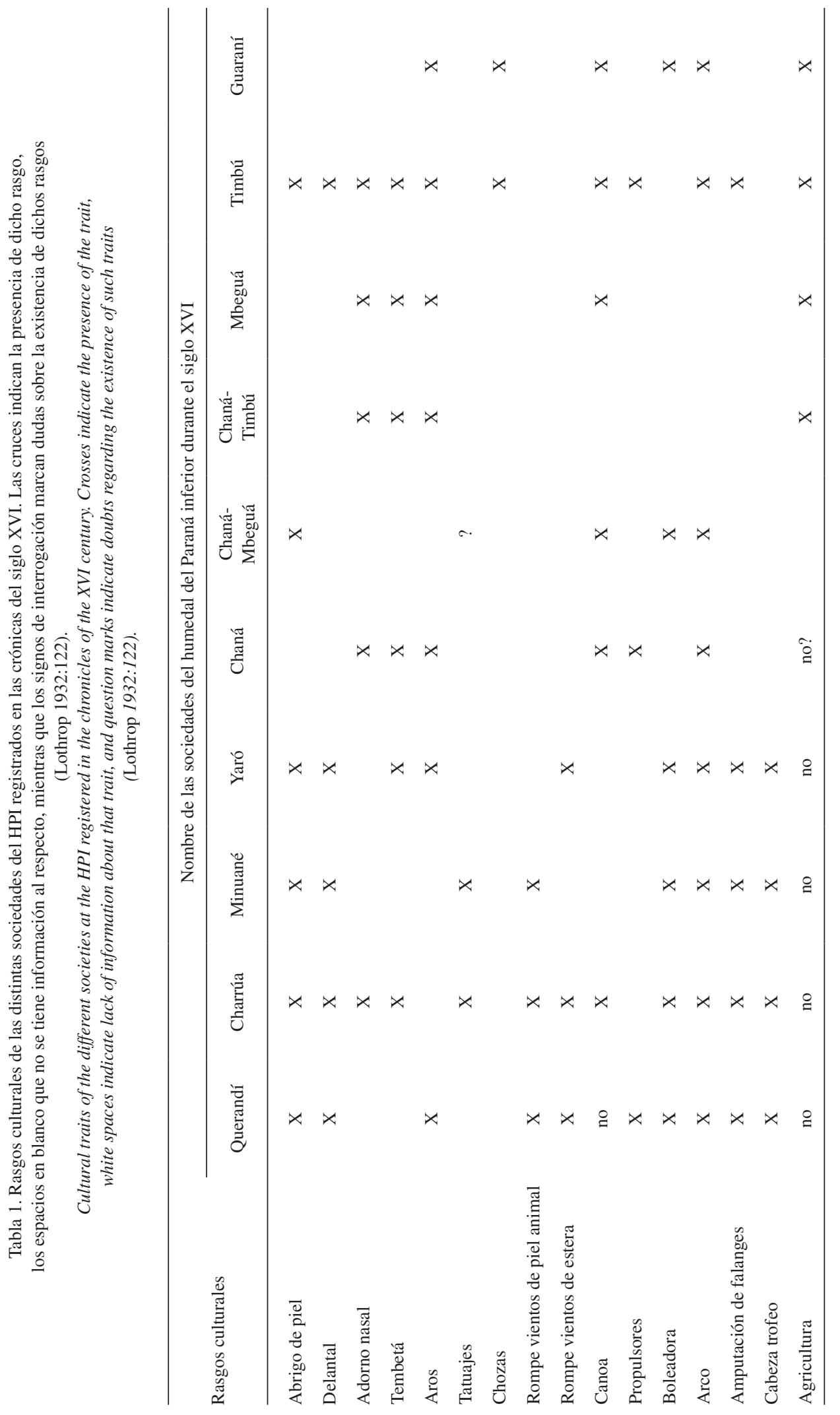


decoración cerámica, adornos personales (Acosta et al. 2010; Loponte 2008), estilos artefactuales (Buc y Silvestre 2010) y costumbres mortuorias (Mazza y Loponte 2012). Estas diferencias fueron interpretadas como el correlato material de la posible coexistencia de varias sociedades, las cuales utilizaban diferentes elementos de su cultura para diferenciarse. Dicha diferenciación habría surgido hacia fines del Holoceno tardío como parte de un proceso de fragmentación espacial y nuclearización de grupos, ocasionado por el aumento demográfico que habría ido ocurriendo a medida que la productividad y estabilidad ambiental aumentaba (Acosta y Loponte 2013; Loponte 2008). Como puede verse, estas inferencias fueron realizadas sobre la base material del registro arqueológico y no así sobre una base biológica. Es decir que no se llevaron a cabo análisis que tengan en cuenta la posible existencia de variaciones biológicas dentro del área de estudio que puedan relacionarse con la presencia de distintas sociedades o poblaciones. Por el contrario, los estudios de variación biológica que analizaron la presencia de diferencias poblacionales han abordado al HPI con fines comparativos para establecer relaciones biológicas con otras regiones y de esta manera elaborar modelos de poblamiento o de relaciones interpoblacionales (p.ej., Bollini et al. 2012; Del Papa 2008; Fabra 2013). Por lo tanto, el objetivo de este trabajo es complementar los estudios mencionados sobre la existencia de diferencias materiales al interior del HPI y evaluar la consecuente hipótesis sobre la posible presencia de diferentes sociedades hacia fines del Holoceno tardío (ca. 2.000-700 años a.p.). Para esto se realizó un análisis de la variación biológica por medio de caracteres epigenéticos. Los rasgos epigenéticos son definidos como formaciones óseas que aparecen circunstancialmente en el cráneo y en el esqueleto poscraneal humano. Como marcadores fenotípicos, constituyen un tipo de variación que puede aportar información valiosa acerca de los procesos de variabilidad biológica, estructuras sociales, fenómenos de perturbación poblacional, flujo génico o mestizaje (González et al. 1999; Hanihara et al. 2003). La heredabilidad de estos rasgos ha permitido su uso como herramienta equivalente a las variables métricas para evaluar distancias biológicas (Fabra 2014; Del Papa 2008; Hanihara et al. 2003).

En este trabajo se estudiaron 39 individuos provenientes de distintos sitios arqueológicos. A los fines de evaluar la variación epigenética craneofacial en la población arqueológica seleccionada, la muestra fue dividida y analizada según dos criterios diferentes. El primero de ellos se basó en una segmentación según criterios arqueológicos en dos grandes unidades geográficas: norte y sur del río Paraná Guazú, brazo más grande del Delta del Paraná, con un ancho variable entre 1 y $2 \mathrm{~km}$. Esta delimitación parte de un estudio arqueológico que indica que este río pudo haber funcionado como un filtro a la transmisión de ciertos aspectos estilísticos de variación tecnológica, los que fueron asociados a la presencia de diferentes sociedades (Buc y Silvestre 2010). El segundo criterio es puramente ambiental. Con fines de estudiar los distintos procesos de adaptación humana, el humedal del Paraná inferior fue segmentado en seis unidades del paisaje según su oferta de recursos y el grado de fragmentación fluvial. Estas unidades son: (1) Delta superior y medio, (2) Delta inferior, (3) Praderas y Sabanas, (4) Praderas del Ibicuy, (5) Planicies inundables y (6) Bajíos Ribereños (Loponte 2008). En este trabajo realizaremos la comparación entre los sectores del Delta inferior y Planicies inundables. Las unidades del paisaje referentes a Praderas del Ibicuy, Bajíos Ribereños y Praderas y Sabanas no fueron incluidas debido a la poca a nula cantidad de individuos disponibles para estos sectores.

\section{Materiales y Métodos}

\section{La muestra}

La muestra se compone de 39 individuos adultos de ambos sexos (29 masculinos y 10 femeninos), provenientes de los siguientes sitios arqueológicos: Cerro Lutz (Acosta y Loponte 2006; Loponte y Acosta 2007), Paranacito (Gatto 19381), Brazo Largo (Gatto 1939), Mazaruca (Lista 1878; Torres 1903), Escuela 31 (Loponte y Acosta 2007), Túmulo 1 del Paraná Guazú, Túmulo 2 del Paraná Guazú, Túmulo del Brazo Gutiérrez (Torres 1911), Arroyo Fredes (Loponte y Acosta 2003/2005) y La Bellaca sitio 1 (Loponte 2008) (Figura 1; Tabla 2).

Los individuos analizados pertenecen al Holoceno Tardío (ca. 2.000-700 años a.p.). Es importante aclarar que esta muestra no incluye a la totalidad de individuos que se recuperaron en los sitios arqueológicos del HPI, sino que representan una submuestra, cuyos criterios de selección estuvieron dados por la integridad de las colecciones museísticas y la posibilidad de registro de 


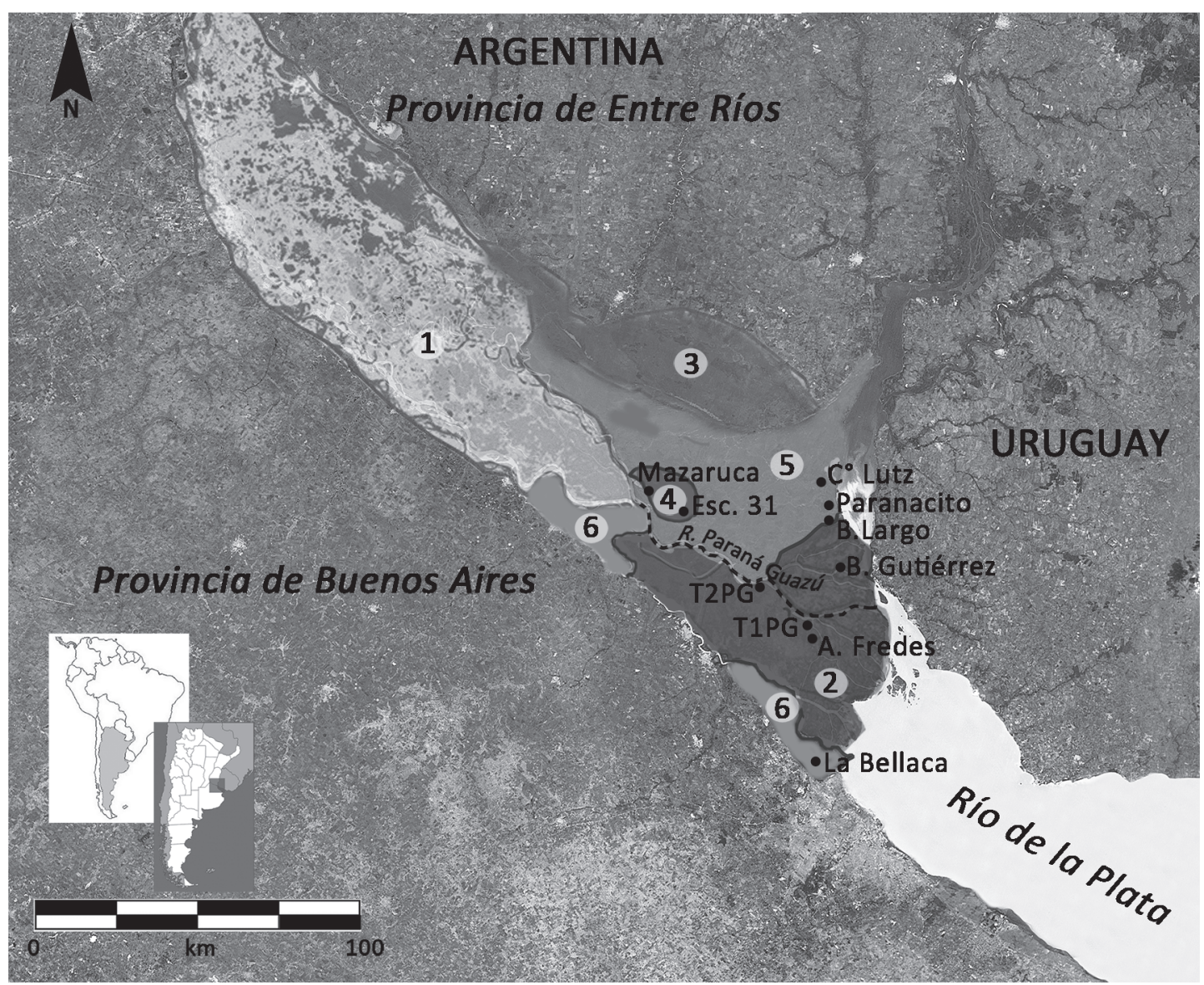

Figura 1. Ubicación geográfica de los sitios arqueológicos mencionados en el texto. La línea punteada delimita los sectores al norte y sur del Paraná Guazú. Los números señalan las distintas unidades del paisaje: (1) Delta superior y medio, (2) Delta inferior, (3) Praderas y sabanas, (4) Praderas del Ibicuy, (5) Planicies inundables y (6) Bajíos Ribereños.

Geographic location of the archaeological sites mentioned in the text. The dotted line demarcatess the north and south areas limit of the Paraná Guazú River. The numbers refers to the different landscape units: (1) Upper and Middle Delta, (2) Lower Delta, (3) Meadow and savannah, (4) Ibicuy meadows, (5) Flood plains and (6) Riverside lowlands.

las variables biológicas. Por lo tanto, las conclusiones a las que se llegan en este estudio están limitadas a la submuestra analizada.

La determinación sexual se realizó mediante el análisis del cráneo y los coxales. Para ello, se implementaron los criterios definidos por Buikstra y Ubelaker (1994) para la glabela, margen supraorbital, proceso mastoides, cresta occipital, eminencia mentoneana del cráneo y escotadura ciática para los coxales, sumado al método de Phenice (1969) para el pubis.

\section{Los sitios arqueológicos}

A continuación se resumen los datos bioarqueológicos disponibles para cada sitio arqueológico que analizamos en este trabajo. A excepción del sitio Arroyo Fredes, que fue atribuido a sociedades horticultoras denominadas Guaraníes, el resto de los sitios arqueológicos pertenecen a sociedades con economía cazadora-recolectora. Esta distinción fue establecida sobre la base del material arqueológico recuperado como así también en relación con la modalidad de las inhumaciones (Acosta 2005; Buc 2010; Loponte 2008; Loponte et al. 2011; Lothrop 1932; Mazza y Loponte 2012; Sacur Silvestre et al. 2013; Vignati 1941).

\section{Paranacito}

Este sitio arqueológico se ubica al sur de la provincia de Entre Ríos y al norte del río Paraná 


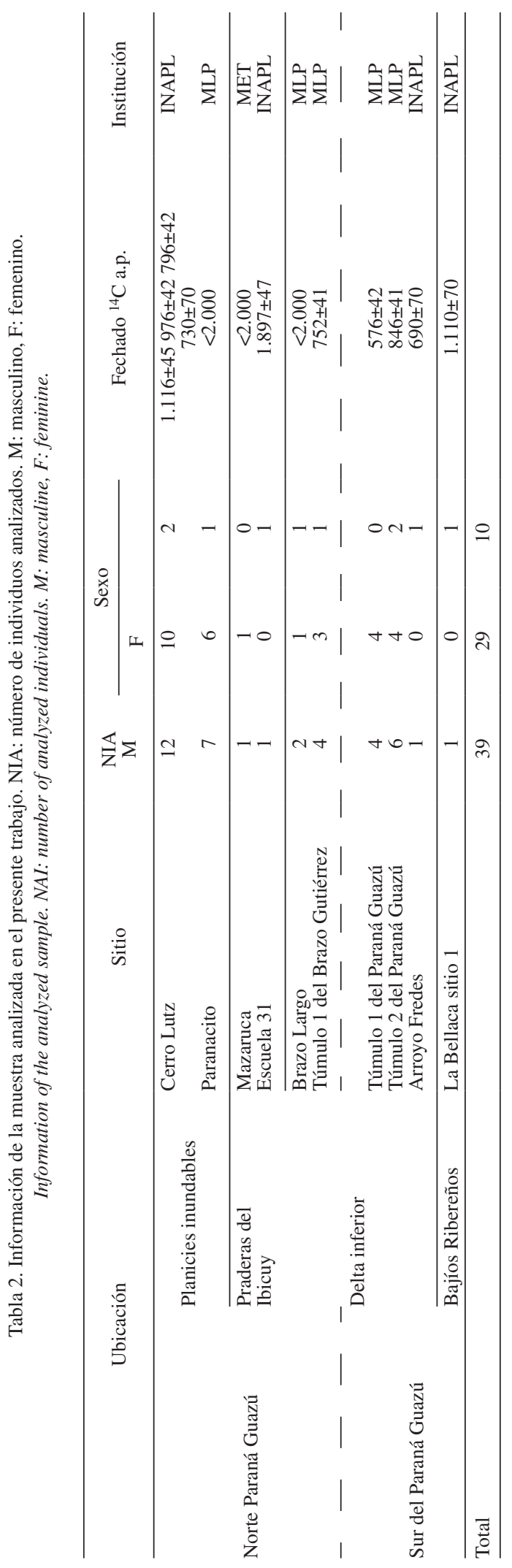

Guazú, perteneciendo a la unidad ambiental de Planicies inundables. Lamentablemente no tenemos información detallada sobre los contextos en que fueron hallados los individuos, como así tampoco del material arqueológico recuperado. Los únicos datos que tenemos son los provenientes de los libros de entrada del Museo Etnográfico de la Universidad de Buenos Aires, en donde se señala que fueron ingresados a la institución por Gatto en 1938. Si bien aún no se tienen fechados radiocarbónicos de este sitio, debido a su ubicación geográfica se puede inferir que su ocupación es posterior a los 2.000 años a.p. (Cavallotto et al. 2004).

La muestra se compone de siete individuos, de los cuales seis son masculinos y uno es femenino (Tabla 2).

\section{Cerro Lutz.}

Se ubica al sur de la provincia de Entre Ríos y al norte del río Paraná Guazú, en el área de Planicies inundables. Este sitio fue excavado en los años 2006 y 2007 por el equipo de investigación, dirigido por Daniel Loponte y Alejandro Acosta. Se trata de un cementerio, cuyos fechados radiocarbónicos lo ubican con posterioridad a los 1.000 años ${ }^{14} \mathrm{C}$ a.p. Se recuperaron alrededor de 40 individuos provenientes tanto de inhumaciones primarias como secundarias, orientados principalmente hacia el suroeste-noreste, aunque se encontró un bajo número de individuos orientados hacia noreste y hacia el este (Acosta y Loponte 2006; Loponte y Acosta 2007; Mazza 2010).

La muestra se compone de 12 individuos, de los que dos son femeninos y 10 son masculinos (Tabla 2).

\section{Mazaruca}

Se encuentra también al sur de la provincia de Entre Ríos, al norte del río Paraná Guazú, en la unidad ambiental de Praderas del Ibicuy. El sitio fue investigado por Lista (1878), quien encontró nueve inhumaciones primarias, en las que solamente pudieron rescatar tres cráneos. La excavación de este sitio fue retomada años más tarde por Torres (1903), quien recuperó un entierro primario correspondiente a un individuo masculino adulto depositado en posición lateral flexionada. Al igual que el sitio Paranacito, los individuos de Mazaruca se ubican dentro de los últimos 2.000 años a.p. Lamentablemente, no sabemos si el individuo incluido en este trabajo 
proviene de las excavaciones de Lista o de Torres. Corresponde a un individuo masculino adulto.

\section{Escuela 31}

Cercano al sitio Mazaruca, fue excavado en el año 2007 por Daniel Loponte y Alejandro Acosta, quienes hallaron una inhumación primaria en posición decúbito ventral, con orientación oeste-este, perteneciente a un individuo de sexo femenino de edad adulta. Recientemente, un fechado radiocarbónico realizado sobre una costilla de dicho individuo arrojó una edad de $1.897 \pm 47$ años ${ }^{14} \mathrm{C}$ a.p. (AA103644; Daniel Loponte comunicación personal 2014).

\section{Brazo Largo}

Este sitio se ubica al norte del río Paraná Guazú sobre el río Brazo Largo, en la unidad perteneciente al Delta inferior. Fue excavado por Gatto (1939), quien recuperó tanto inhumaciones primarias como secundarias. Lamentablemente, no podemos saber a qué inhumación se refieren los dos individuos analizados en este trabajo, de la misma forma en que desconocemos sus orientaciones y posiciones. Se trata de un individuo femenino y un masculino, ambos adultos (Tabla 2).

\section{Túmulo 1 y 2 del Paraná Guazú y Túmulo del Brazo Gutiérrez.}

Estos sitios se ubican en el sector insular del Delta inferior, sobre los ríos que llevan el nombre de cada sitio. Mientras que Túmulo 1 y 2 del Paraná Guazú se ubican al sur de dicho río, Túmulo del Brazo Gutiérrez lo hace al norte. Todos ellos fueron investigados por Torres (1911), dando como resultado el descubrimiento de al menos un centenar de individuos. Desafortunadamente, este autor no especifica la modalidad de inhumación para ninguno de los entierros recuperados y solo menciona, para algunos casos, la posición del cráneo. A su vez, si bien tampoco L. M. Torres ofrece información escrita sobre las orientaciones de las inhumaciones, las mismas pueden ser inferidas a partir de los croquis publicados. Sobre la base de esta información puede determinarse que predomina el eje oeste-este por sobre el noreste-sudoeste, sudoeste-noreste y esteoeste (Mazza y Loponte 2012; cfr. Torres 1911). Un fechado sobre un individuo del sitio Túmulo 1 del Paraná Guazú arrojó una antigüedad de 576 \pm 42 años ${ }^{14} \mathrm{C}$ a.p. Mientras que el sitio Túmulo 2 fue fechado en $690 \pm 70$ años ${ }^{14} \mathrm{C}$ a.p. (Bernal 2008).

En este trabajo se incluyeron 14 individuos provenientes de los tres sitios (11 masculinos y tres femeninos, Tabla 2).

\section{La Bellaca sitio 1}

Este sitio se ubica en la provincia de Buenos Aires, al sur del río Paraná Guazú, en el área de Bajíos Ribereños. Lamentablemente, la inhumación fue extraída por coleccionistas locales y, por ende, se desconoce la modalidad de entierro como así también su posición y orientación. Corresponde a un individuo femenino adulto. Un hueso de mamífero perteneciente al sitio fue fechado en $1.110 \pm 70$ años ${ }^{14}$ C a.p. (Loponte 2008).

\section{Arroyo Fredes}

Se ubica al sur del Paraná Guazú, en la zona del Delta inferior, provincia de Buenos Aires. Fue excavado originalmente por Gaggero en el primer cuarto del siglo XX, mientras que los restos humanos recuperados fueron estudiados por Vignati (1941). En este sitio se extrajeron inhumaciones contenidas en urnas funerarias, atribuidas a grupos Guaraníes. Un fechado sobre uno de los individuos recuperados arrojó una edad de $690 \pm 70$ años ${ }^{14} \mathrm{C}$ a.p. (Loponte y Acosta 2003-2005). En excavaciones recientes realizadas por Loponte y Acosta se extrajeron dos entierros primarios en decúbito dorsal flexionado, con orientación norte-sur. Uno de estos individuos integra la muestra analizada en este trabajo, correspondiente a un femenino adulto. Un nuevo fechado sobre material faunístico proveniente del sitio arrojó una edad de $402 \pm 40$ años ${ }^{14} \mathrm{C}$ a.p. y otro, realizado sobre uno de los dos entierros primarios mencionados, una edad de $370 \pm 50$ años ${ }^{14} \mathrm{C}$ a.p. (Loponte et al. 2011).

\section{Las variables}

\section{Análisis de la variabilidad epigenética craneofacial}

En el presente trabajo se relevaron 19 rasgos epigenéticos craneales: sutura metópica, escotadura supraorbital, foramen supraorbital, sutura infraorbital, forámina infraorbital múltiple, forámina cigomático facial, foramen parietal, wormianos epiptérico, coronal, 
sagital, apical, lambdoide, astérico, mastoideo y supramastoideo, hueso inca, canal condileo, canal del hipogloso dividido y foramen mastoideo (Buisktra y Ubelaker 1994). En cada caso se codificó con 0 o 1 la ausencia o presencia de estos rasgos.

\section{Análisis estadísticos exploratorios}

En primer lugar se realizó un análisis de Chicuadrado $(p<0,05)$ para evaluar la asociación de los rasgos epigenéticos con el sexo. Solamente dos rasgos se vieron influidos por esta variable (escotadura supraorbital y wormiano lambdoide), y fueron eliminados de los análisis subsiguientes. Posteriormente, se calculó el coeficiente de comparación simple (simple matching coefficient; Sneath y Sokal 1973) y las distancias genéticas fueron interpretadas a través del análisis de conglomerados, utilizando la técnica de agrupamiento por pares mediante ligamiento promedio no ponderado (UPGMA). Luego, se realizó un análisis de componentes principales con una matriz de 15 rasgos epigenéticos, eliminando aquellos que tenían valor de cero en cada individuo. El objetivo de estos análisis es explorar los patrones de agrupación de los individuos en función de las distancias biológicas calculadas sobre los rasgos epigenéticos seleccionados, sin diferenciar la muestra por regiones o ubicación geográfica.

\section{Análisis confirmatorios}

Se decidió aplicar la técnica de análisis de permutación de respuesta múltiple (MRPP) para poner a prueba la homogeneidad o heterogeneidad biológica de la población estudiada, considerando variables como el sexo (femenino o masculino) o la segmentación geográfica (norte y sur del Paraná Guazú por un lado y Delta inferior y Planicies inundables por el otro) para su agrupamiento.

El MRPP es un procedimiento no paramétrico, equivalente al análisis discriminante o al ANOVA de una vía, que permite poner a prueba la hipótesis de distribución no aleatoria de la variación biológica. Como ventaja adicional, permite evaluar la homogeneidad interna de cada grupo a partir del cálculo de distancias promedio entre casos dentro de estos. El método parte del cálculo de las distancias entre todos los distintos pares de puntos y luego del promedio ponderado o delta. El promedio ponderado observado de estas distancias (observed delta) se compara con la distancia que habría si dichos grupos hubieran sido definidos aleatoriamente (expected delta). La homogeneidad relativa de cada grupo se evalúa en relación con los valores de delta. Promedios ponderados bajos indican una tendencia al agrupamiento, mientras que valores altos indican falta de agrupamiento (Zimmerman et al. 1985). Se considera significativo un valor de p igual o inferior a 0,05 .

\section{Resultados}

\section{Análisis exploratorios}

Respecto de la prevalencia de los rasgos epigenéticos de los individuos relevados, considerando la división en norte y sur con referencia al río Paraná Guazú, la mayoría de los rasgos presenta frecuencias mayores al sur del Paraná Guazú, con excepción de la foramina infraorbital múltiple y del hueso wormiano epiptérico, que solo se encuentran presentes en el sector norte (Figura 2A). Estos rasgos coinciden con aquellos presentes si se hace una división entre las unidades del paisaje pertenecientes al Delta inferior y a Planicies inundables (Figura 2B). Con una pequeña diferencia de porcentajes, los valores referentes al sur del Paraná Guazú son concordantes con los encontrados en el Delta inferior, mientras que aquellos que prevalecen al norte del Paraná Guazú coinciden con los de Planicies inundables. Otros rasgos se encuentran ausentes en toda la muestra, como la sutura metópica, los huesos wormianos coronal, sagital e Inca.

El análisis de conglomerados muestra tres conjuntos claramente diferenciados a un coeficiente de 0,73 , e individuos que se alejan de estas agrupaciones a mayores valores de distancia (individuos número 33 de Túmulo 1 del Paraná Guazú, 21 de Cerro Lutz y 15.904 del sitio Paranacito) (Figura 3A). Si bien todos los conglomerados están integrados por muestras procedentes de los distintos sitios arqueológicos relevados, se observa que los individuos del norte del Paraná Guazú se agrupan principalmente en el conglomerado denominado arbitrariamente 3 , representando el $74,07 \%$ del total de individuos ubicados al norte de dicho río $(\mathrm{n}=20$ de un total de 27 individuos). Si bien se trata de un análisis exploratorio, es interesante considerar esta tendencia o patrón de agrupación subregional. No se observan agrupaciones en relación con las unidades del paisaje.

El análisis de componentes principales muestra cierta agrupación geográfica. Considerando la 


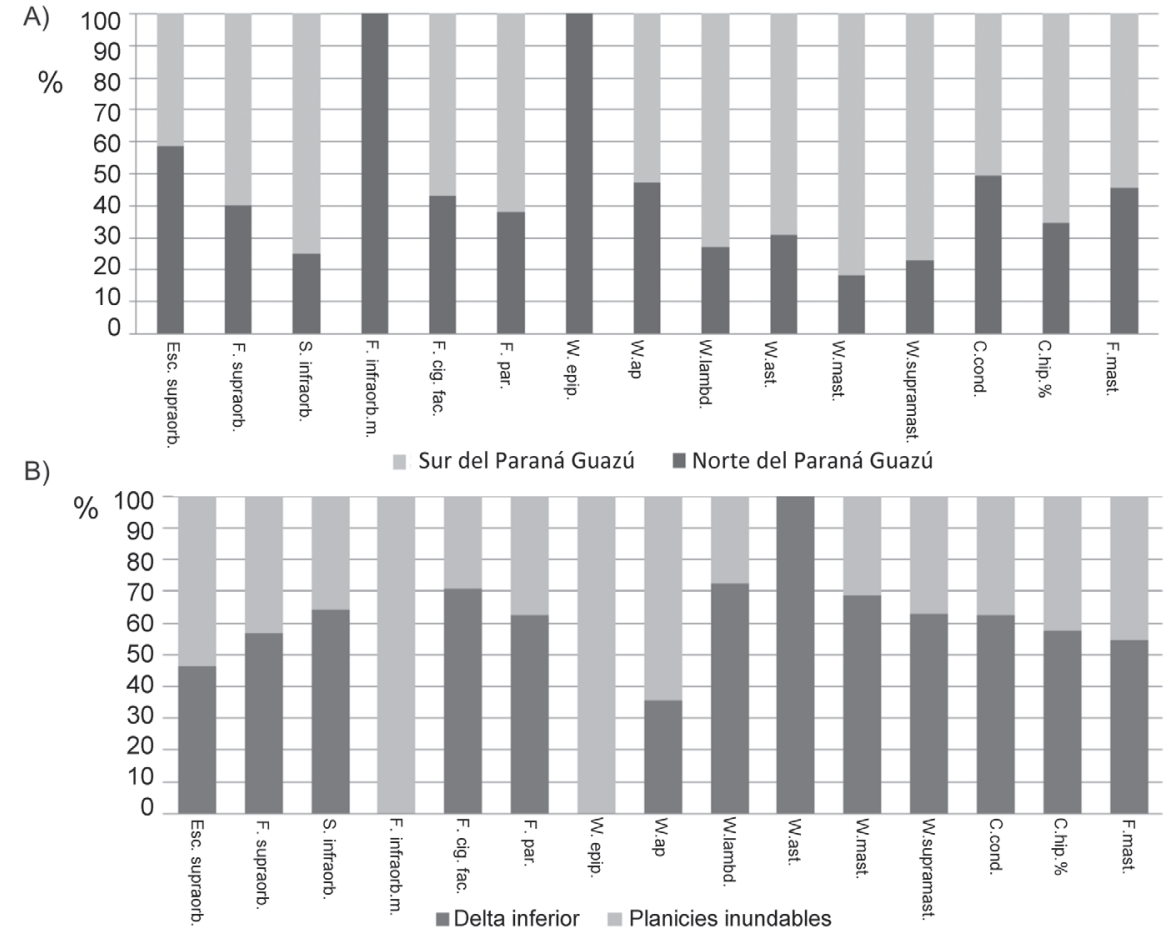

Figura 2. Prevalencias de rasgos epigenéticos considerando la ubicación respecto del río Paraná Guazú (A) y las unidades del paisaje Delta inferior y Planicies inundables (B).

Prevalences of epigenetic traits, considering the location in relation to the Paraná Guazú River (A) and the landscape units $(B)$.

ubicación de las muestras respecto del primer componente, que explica solo el $17,23 \%$ de la variación total de la muestra, a la derecha se ubican la mayoría de los individuos correspondientes a los conjuntos definidos como 1 y 2 en el análisis de conglomerados, aunque con la inclusión de los individuos del sitio Brazo Largo. En cambio, hacia la izquierda del mismo componente, se agrupan la mayoría de los individuos del conjunto número 3. Nuevamente el individuo número 33 del sitio Túmulo 1 del Paraná Guazú se aleja claramente del conjunto, mientras que el individuo $21 \mathrm{del}$ sitio Cerro Lutz y el 15904 de Paranacito aparecen integrados al resto de los individuos analizados (Figura 3B). Las variables que más contribuyen a este agrupamiento son, con peso positivo, el wormiano mastoideo y el canal condileo en el primer componente y los wormianos apical y supramastoideo en el segundo componente. No se observan correspondencias con las unidades del paisaje.

Si bien hay ciertas coincidencias entre el análisis de componentes principales con el análisis de conglomerados, hay que tener en cuenta que, comparativamente, los primeros dos componentes principales explican solo una pequeña fracción de variación, cubriendo apenas el $31,44 \%$ del total, por lo que esta técnica de ordenación no parece ser la indicada para representar de manera fidedigna las similitudes relativas entre las muestras.

\section{Análisis confirmatorio}

Finalmente, se decidió realizar un análisis de permutación de respuesta múltiple (MRPP), en este caso para poner a prueba la existencia de un correlato entre las diferencias observadas, considerando la división del norte y sur respecto del río Paraná Guazú, las dos unidades del paisaje (Delta inferior y Planicies inundables), el sexo de los individuos y la composición biológica de las poblaciones. En este caso, los resultados fueron significativos únicamente para la segmentación por unidad del paisaje. La agrupación geográfica con referencia al Paraná Guazú y el sexo de los individuos no fueron significativas (Tabla 3). 

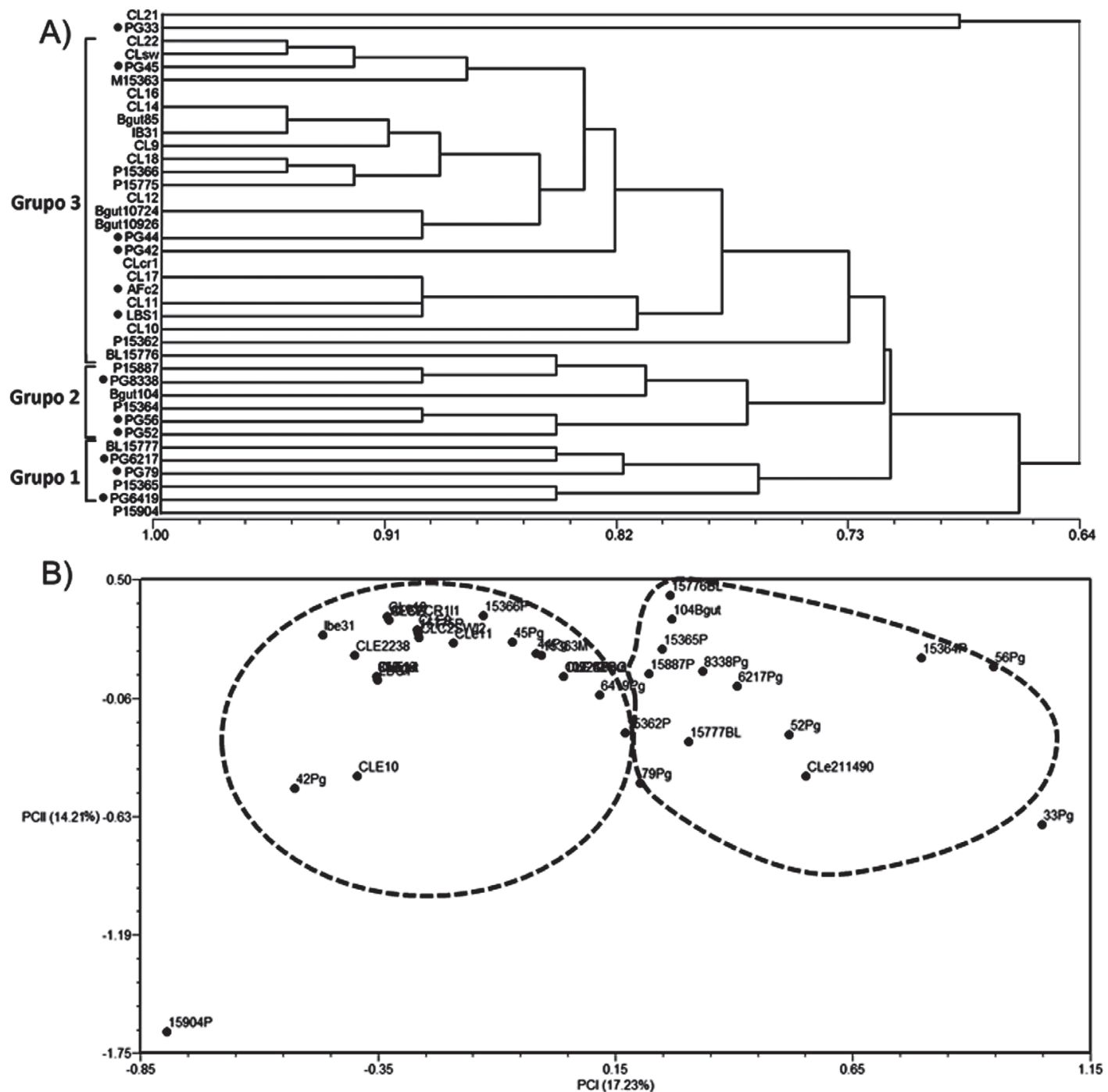

Figura 3. Análisis de conglomerados (A) y análisis de componentes principales (B) a escala intrapoblacional. En el análisis de conglomerados, el círculo señala a los individuos procedentes del sur del Paraná Guazú. Referencias: CL: Cerro Lutz; BL: Brazo Largo; P: Paranacito; IB: Escuela 31, Pg: Túmulo 1 y 2 del Paraná Guazú, M: Mazaruca, LB: La Bellaca sitio 1; Bgut: Túmulo del Brazo Gutiérrez; AF: Arroyo Fredes.

Cluster analysis $(A)$ and principal components analysis $(B)$ at intrapoblational scale. In the cluster analysis the circles indicates the individuals from the south of the Paraná Guazú River. CL: Cerro Lutz; BL: Brazo Largo; P: Paranacito; IB: Escuela 31, Pg: Túmulo 1 y 2 del Paraná Guazú, M: Mazaruca, LB: La Bellaca sitio 1; Bgut: Túmulo del Brazo Gutiérrez; AF: Arroyo Fredes.

\section{Discusión}

La existencia de límites sociales ha sido abordada arqueológicamente dentro de sociedades cazadorasrecolectoras a partir del estudio de ciertos rasgos culturales como ser el estilo/decoración cerámica, el uso de adornos personales o tatuajes corporales (Graves 1994; Loponte 2008; Loponte et al. 2012; Palavecino 1922; Rice 1999; Rodríguez y Ceruti
1999; Serrano 1972; Stark 1998; Torres 1911) y en la presencia de distintos aspectos mortuorios (Beck 1995; Fisher 1995; Mazza y Loponte 2012). Estas investigaciones analizan los distintos patrones culturales de varios sitios en una región determinada, buscando variaciones en el registro material que permitan hipotetizar sobre la existencia de límites sociales. En este sentido, los distintos grupos étnicos utilizarían su cultura material como un medio para 
Tabla 3. Resultado del análisis de MRPP. Valores de distancia promedio dentro de grupos, considerando las agrupaciones geográficas y el sexo de los individuos (distancia euclidiana cuadrada).

MRPP analysis results. Values of mean distances withingroups, considering geographic location and sex of the individuals (Squared Euclidean distance).

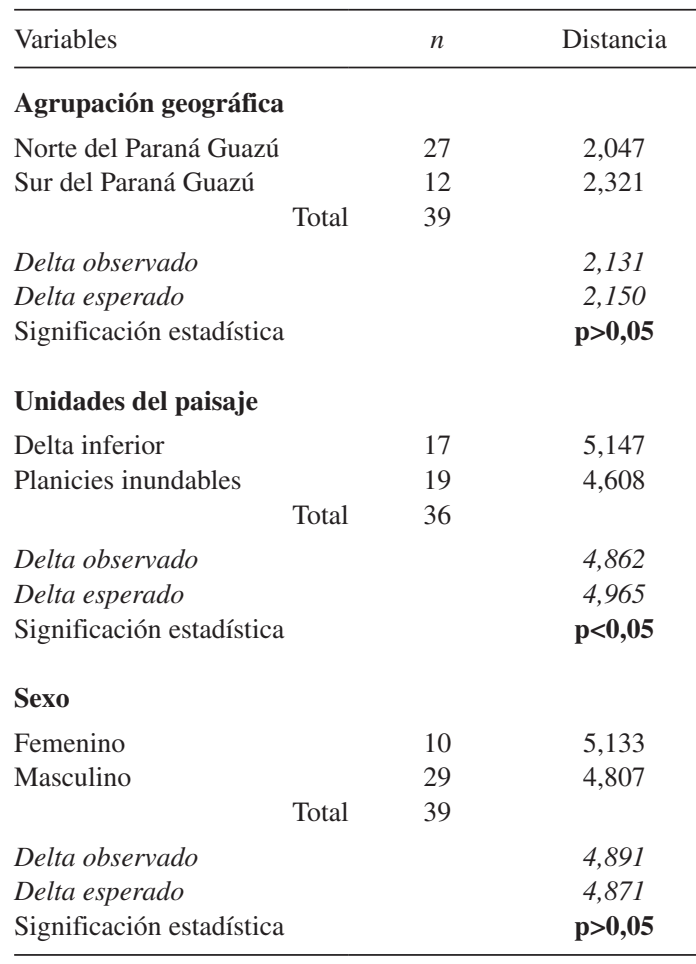

simbolizar distinciones sociales y pertenencia étnica frente a otros grupos fuera de su territorio (Hodder 1985). Estas barreras quedarían demostradas por ciertas diferencias a nivel artefactual (forma, decoración) y también a nivel mortuorio. Pero estos límites étnicos no serían impermeables sino que, por el contrario, el intercambio de información y de bienes sería posible. Esto último incentivaría a que existan similitudes en el estilo artefactual y posiblemente también en las prácticas mortuorias (Beck 1995).

En lo que concierne al HPI, la presencia de límites sociales fue inferida a través de ciertas diferencias del registro material entre distintas subdivisiones internas a la región (Acosta et al. 2010; Buc y Silvestre 2010; Loponte 2008) y no así a través de variables biológicas. El presente estudio permitió evaluar la tendencia hacia una agrupación regional diferenciada, entre los individuos procedentes, por un lado, del Delta inferior y de Planicies inundables y, por el otro, del norte y sur del río Paraná Guazú. Los análisis de las prevalencias de los rasgos epigenéticos muestran un patrón similar para ambas divisiones geográficas. Tanto en el norte del Paraná Guazú como específicamente en su subregión denominada Planicies inundables predominan la foramina infraorbital múltiple y el hueso wormiano epiptérico. En cambio, al sur del Paraná Guazú y en su subregión Delta inferior, ambas variables se encuentran completamente ausentes. A pesar de estas diferencias, los análisis de permutación de respuesta múltiple solo corroboraron las existentes entre el Delta inferior y las Planicies inundables. Posiblemente esto se deba a que la división respecto del norte y sur del Paraná Guazú presente una mayor homogeneidad interna en ambos subconjuntos al incluir otras subregiones como ser Bajíos Ribereños y Praderas del Ibicuy, lo cual derive en que no se observen diferencias significativas entre ellos. Esto es lo que claramente se puede observar en los análisis de conglomerados y de componentes principales, donde si bien hay cierta agrupación siguiendo un eje norte-sur del Paraná Guazú, se observa al interior de cada grupo la presencia de individuos que no siguen dicha delimitación geográfica, provocando cierta confusión en los datos y, por ende, desestimando la aplicación de ambos análisis. Esperamos en un futuro cercano haber aumentado el número de individuos provenientes de las zonas de Bajíos Ribereños y Praderas del Ibicuy, con fines de poder evaluar si estas dos subregiones contribuyen a la homogeneización entre el norte y sur del Paraná Guazú o, si por el contrario, los resultados obtenidos son producto del tamaño de muestreo actual.

Por el momento, estos resultados indican que a pesar de haber ciertas diferencias en el registro artefactual con referencia al norte y sur del Paraná Guazú (Buc y Silvestre 2010), las mismas no se condicen con los resultados biológicos y por lo tanto posiblemente no estén demarcando la presencia de diferentes sociedades. Lo contrario sucede con las subregiones del Delta inferior y de Planicies inundables donde, según los resultados significativos obtenidos, es posible inferir la presencia de dos sociedades diferentes. Si bien todavía no se realizaron estudios comparativos exhaustivos sobre las similitudes o diferencias en el registro material de estas dos unidades del paisaje que nos permita correlacionar sus resultados con los observados en este trabajo, podemos mencionar el análisis sobre la variabilidad regional de las prácticas mortuorias de sociedades cazadoras-recolectoras realizado por Mazza y 
Loponte (2012), quienes incluyen a las unidades del paisaje mencionadas. En dicho trabajo se observaron diferencias estadísticamente significativas entre los patrones mortuorios de los sectores continentales (Praderas del Ibicuy, Bajíos Ribereños) y aquellos denominados insulares (Delta superior e inferior y Planicies inundables). En el primero predominan los entierros primarios, mientras que en el sector insular hay una mayoría de inhumaciones secundarias (Mazza y Loponte 2012). Lamentablemente, la información mortuoria disponible para varios de los sitios arqueológicos e individuos que incluimos en este trabajo es muy escasa como para realizar comparaciones entre sectores insulares y continentales, sus prácticas mortuorias y la variación biológica craneal para evaluar correspondencias o diferencias entre ellas (ver descripción de los sitios en el apartado de Materiales). Sin embargo, podemos observar ciertos patrones con las inhumaciones provenientes del Delta inferior y Planicies inundables. En este sentido, los entierros primarios provenientes de ambas unidades ambientales y las inhumaciones secundarias del sector de Planicies inundables difieren en las frecuencias de los rasgos epigenéticos observados, donde entre los primeros están presentes la foramina infraorbital múltiple y el hueso wormiano epiptérico, mientras que entre los entierros secundarios estas variables están completamente ausentes (Figura 4). A su vez, el análisis de MRPP arrojó resultados significativos exclusivamente en el caso de las modalidades de inhumación $(\mathrm{p}<0,05)$, no así en las posiciones u orientaciones de los individuos (Tabla 4). Es importante
Tabla 4. Resultado del análisis de MRPP. Valores de distancia promedio dentro de grupos, considerando las modalidades de entierro, posiciones y orientaciones de sitios arqueológicos del Delta inferior y Planicies inundables (distancia euclidiana cuadrada).

MRPP analysis results. Values of mean distances withingroups, considering burial types, positions and orientations from archaeological sites of the Lower Delta and Flood plains (Squared Euclidean distance).

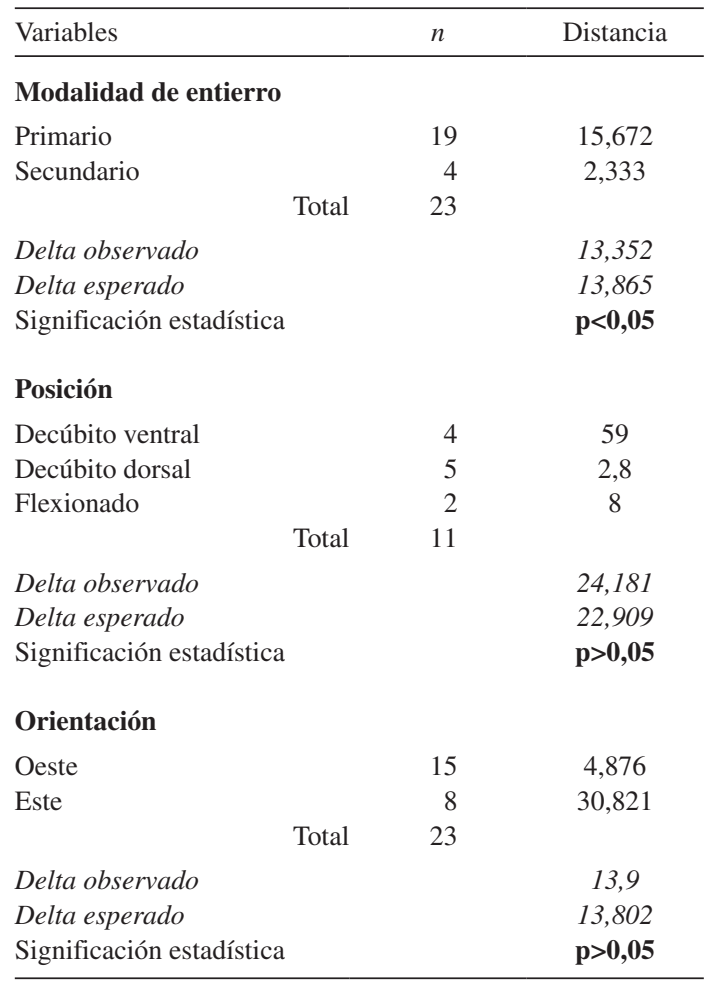

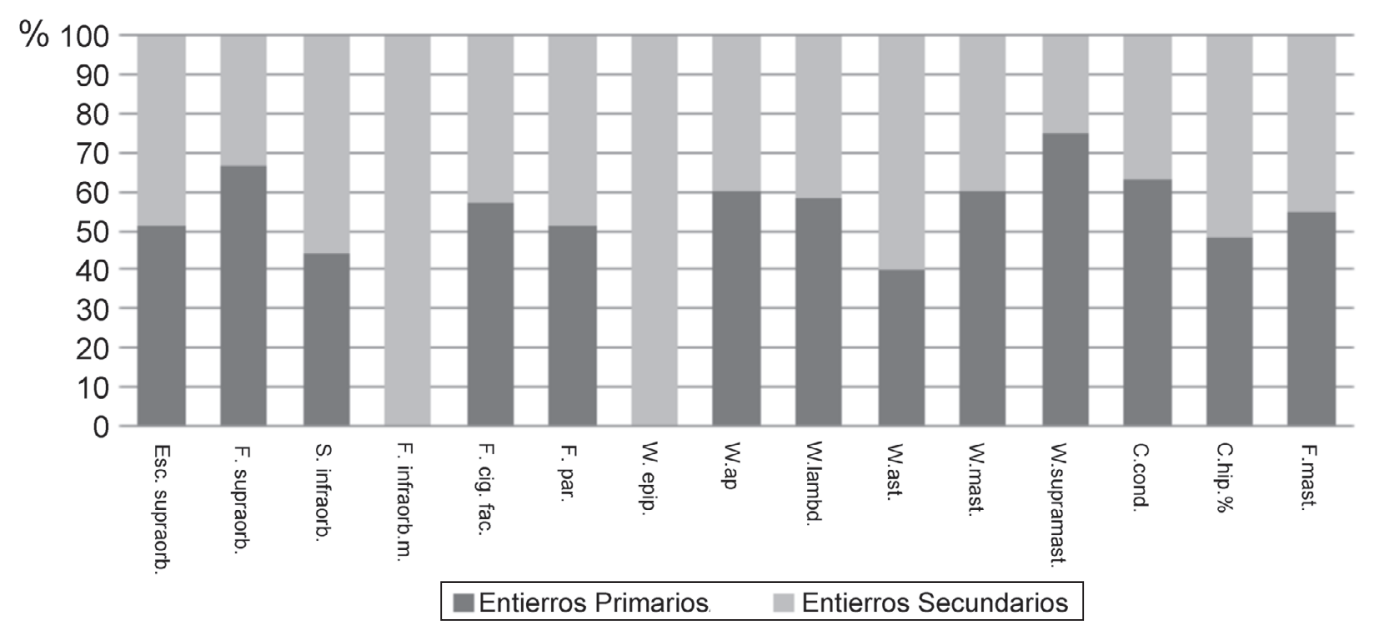

Figura 4. Prevalencias de rasgos epigenéticos según modalidad de inhumación.

Prevalences of epigenetic traits regarding types of burial. 
mencionar que estos resultados posiblemente sean dependientes del tamaño de muestra como así también de su información asociada disponible. Sería interesante obtener a futuro inhumaciones secundarias con presencia de cráneos provenientes de otros sectores, fundamentalmente del Delta inferior, para poder evaluar si difieren o no de aquellos de las Planicies inundables.

\section{Conclusiones}

Las diferencias biológicas observadas en este trabajo en relación con la manifestación de ciertos rasgos epigenéticos craneales, sugieren la existencia de diferentes sociedades asentadas en el HPI, más precisamente en los sectores de Planicies inundables y Delta inferior. A su vez, la correlación de estos rasgos con las modalidades de inhumación abre nuevos interrogantes sobre las prácticas mortuorias de estas sociedades, las que necesitan de un número mayor de muestras para poder ser respondidas.

Estos resultados deben ser tomados como el puntapié que permita, frente a la generación de nuevos datos y materiales provenientes de nuevas excavaciones, continuar analizando diferencias intrarregionales. La adición de más muestras nos permitirá contrastar las tendencias aquí expuestas como así también comparar aquellas unidades del paisaje que no pudieron ser incorporadas en este análisis.

Consideramos a estos resultados, si bien preliminares en función del bajo n muestral, como un gran avance para la arqueología regional, ya que es la primera vez que la existencia de diferencias biológicas pueden ser interpretadas como la coexistencia de diferentes sociedades mediante análisis que exceden la comparación intrarregional de la variabilidad artefactual.

Agradecimientos: A Daniel Loponte, Alejandro Acosta, Mirta Bonnin, Museo Municipal de Historia de San Rafael, Museo de Ciencias Naturales y Antropológicas "Emilio y Duncan Wagner", Miriam Tarragó, Héctor Pucciarelli, Mónica Sans, Leonel Pérez, Arturo Toscano, Alfredo Casaravilla Nodar. A los tres evaluadores anónimos cuyos comentarios y sugerencias mejoraron este artículo. Esta investigación forma parte de los proyectos PIP 2011-2013 -114 20100100164 (Dir.: Dra. Mariana Fabra), PIP 11220110100565 y PICT 2011-02035 (Dir. Dres. Alejandro Acosta y Daniel Loponte). Finalmente, las autoras agradecen el asesoramiento brindado por Darío Demarchi para el análisis estadístico de los datos.

\section{Referencias Citadas}

Acosta, A. 2005. Zooarqueología de Cazadores-Recolectores del Extremo Nororiental de la Provincia de Buenos Aires (Humedal del Río Paraná Inferior, Región Pampeana, Argentina). Tesis de doctorado, Facultad de Ciencias Naturales y Museo, Universidad Nacional de La Plata, La Plata.

Acosta, A., S. Escudero, R. Feuillet Terzaghi, D. Loponte y L. Pérez Jimeno 2010. Conectando registros: Variabilidad arqueológica en la cuenca del Paraná. En Mamül Mapu: Pasado y Presente desde la Arqueología Pampeana, editado por M. Berón, L. Luna, M. Bonomo, C. Montalvo, C. Aranda y M. Carrera Aizpitarte, pp. 241-252. Libros del Espinillo, Buenos Aires.

Acosta, A. y D. Loponte 2006. Informe sobre las investigaciones realizadas en el sitio arqueológico "Cerro Lutz", Provincia de Entre Ríos (Humedal del Paraná Inferior). Secretaría de Cultura de la Nación, Instituto Nacional de Antropología y Pensamiento Latinoamericano, Buenos Aires.

Acosta, A. y D. Loponte 2013. Complejidad social y estrategias de subsistencia de las poblaciones cazadoras-recolectoras del humedal del Paraná inferior. Cuadernos del Instituto Nacional de Antropología y Pensamiento Latinoamericano. Series Especiales 1(4):60-74.
Beck, A.L. 1995. Regional cults and ethnic boundaries in "Southern Hopewell". En Regional Approaches to Mortuary Analysis, editado por A. Beck, pp. 167-184. Plenun Press, New York.

Bernal, V. 2008. Procesos de Diferenciación Biológica entre Poblaciones Humanas del Holoceno Tardío de Patagonia. Una Aproximación desde la Variación Métrica Dental. Tesis de doctorado, Facultad de Ciencias Naturales y Museo, Universidad Nacional de La Plata, La Plata.

Bollini, G., J. Atencio y S. Colantonio 2012. El hipocono en poblaciones aborígenes de Argentina. Un análisis comparativo poblacional. Revista Argentina de Antropología Biológica 14:57-64.

Buc, N. 2010. Nuevos aportes a la tecnología ósea de la cuenca inferior del río Paraná (Bajíos Ribereños Meridionales, Argentina). Arqueología Iberoamericana 8:21-51.

Buc, N. y R. Silvestre 2010. Distribución de artefactos líticos y óseos en el humedal del Paraná inferior. Relacionando conjuntos del norte y sur del Paraná Guazú. En Arqueología de Cazadores Recolectores en la Cuenca del Plata, editado por G. Cocco y M.R. Feuillet Terzaghi, pp. 171-188. Centro de Estudios Hipanoamericanos, Santa Fe. 
Buikstra, J. y D. Ubelaker 1994. Standards for Data Collection from Human Skeletal Remains. Arkansas Archeological Survey, Arkansas.

Cavallotto, J., R. Violante y G. Parker 2004. Sea-level fluctuations during the last 8600 years in the la Plata river (Argentina). Quaternary International 114:155-165.

Del Papa, M. 2008. Estructuración espacial de la variación biológica humana en la República Argentina durante el Holoceno tardío final a través de los rasgos epigenéticos creaneofaciales. Revista Argentina de Antropología Biológica 10:21-41.

Fabra, M. 2013. Variación epigenética craneofacial y dinámica evolutiva de poblaciones humanas del norte de la región pampeana durante el Holoceno Tardío. Cuadernos del Instituto Nacional de Pensamiento Latinoamericano Series Especiales 1:74-86.

Fabra, M. 2014. Historia de las Poblaciones Prehispánicas del sector austral de las Sierras Pampeanas: Variabilidad Morfológica y Modelos Arqueológicos. Publicaciones de la Secretaría de Ciencia y Tecnología, FFyH, Universidad Nacional de Córdoba, Córdoba.

Fisher, G. 2005. Kingdom and community in early anglo-saxon eastern England. En Regional Approaches to Mortuary Analysis, editado por A. Beck, pp. 147-163. Plenun Press, New York.

Gatto, S. 1939. El paradero-cementerio de Brazo Largo (Delta del Paraná). Physis XVI:365-376.

González, R., J.R. Zavatti y S.L. Dahinten 1999. Influencia de los factores sexo, edad y deformación artificial sobre la variación discontinua en cazadores-recolectores del Noreste de la Patagonia. Revista Argentina de Antropología Biológica 2:201-210.

Graves, M.W. 1994. Community boundaries in the late prehistoric Puebloan society: Kalinga ethnoarchaeology as a model for the southwest production and exchange of pottery. En The Ancient Southwestern Community: Models and Method for the Study of the Prehistoric Social Organization, editado por W.H. Willis y R.D. Leonard, pp. 149-169. University of New Mexico Press, Albuquerque.

Hanihara T., H. Ishida y Y. Dodo 2003. Characterization of biological diversity through analysis of discrete cranial traits. American Journal of Physical Anthropology 121:241-251.

Hodder, I. 1985. Boundaries as strategies: An ethnoarchaeological study. En The Archaeology of Frontiers and Boundaries, editado por S.W. Green y S.M. Perlman, pp. 141-159. Academic Press, New York.

Lista, R. 1878. Cimetières et paraderos minuanes de la province d'Entre-Rios. Revue d'Anthropologie 1:23-32.

Lopes de Sousa, P. 1927. Diario de Navegação da Armada que foi a Terra do Brasil em 1530, Comissão Brasileira dos Centenarios portugueses, Río de Janeiro.

Loponte, D. 2008. Arqueología del Humedal del Paraná Inferior. En Arqueología de la Cuenca del Plata. Serie Monográfica 1, editado por A. Acosta y D. Loponte. Ediciones del Riel, Buenos Aires.

Loponte, D. y A. Acosta 2003/2005. Nuevas perspectivas para la arqueología "Guaranî” en el Humedal del Paraná Inferior y Río de la Plata. Cuadernos del Instituto Nacional de Antropología y Pensamiento Latinoamericano 20:179-197.
Loponte, D. y A. Acosta 2007. Informe sobre las investigaciones arqueológicas realizadas en el Sur de la provincia de Entre Ríos. Segunda Temporada (2006). Secretaría de Cultura de la Nación, Instituto Nacional de Antropología y Pensamiento Latinoamericano, Buenos Aires.

Loponte, D., A. Acosta, I. Capparelli y M. Pérez 2011. La arqueología guaraní en el extremo meridional de la cuenca del Plata. En Arqueología Tupíguaraní, editado por L. Loponte y A. Acosta, pp. 111-154. Instituto Nacional de Antropología y Pensamiento Latinoamericano, Buenos Aires.

Loponte, D., A. Acosta y L. Mucciolo 2012. Contribución a la arqueología del delta del Paraná: El nivel acerámico del sitio Isla Lechiguanas 1. Comechingonia 16:229-268.

Lothrop, S. 1932. Indians of the Parana Delta, Argentina. Annals of the New York Academy of Scienses 33:77-232.

Lozano, P. 1874. Historia de la Conquista del Paraguay, Río de la Plata y Tucumán.Tomo I, Imprenta Popular, Buenos Aires.

Mazza, B. 2010. Cerro Lutz: Aproximaciones al estudio de las prácticas mortuorias de las sociedades cazadoras-recolectoras del humedal del Paraná inferior. La Zaranda de Ideas 6:91-116.

Mazza, B. y D. Loponte 2012. Las prácticas mortuorias en el humedal del Paraná inferior. Arqueología Iberoamericana 13:3-21.

Oviedo, F. y G. Valdés 1944. Historia General y Natural de las Indias. Islas y Tierra-Firme del Mar Océano Vol. 7. Editorial Guarania, Asunción del Paraguay.

Palavecino, E. 1922. Observaciones etnográficas sobre tribus aborígenes del Chaco Occidental. GAEA II:187-209.

Phenice, T. 1969. A new developed visual method of sexing in the os pubis. American Journal of Physical Anthropology 30:297-301.

Rice, P. 1999. On the origins of pottery. Journal of Archaeological Method and Theory 6:1-54.

Rodríguez, J.A. y N.A. Ceruti 1999. Las tierras bajas del nordeste y litoral mesopotámico. En Nueva Historia de la Nación Argentina tomo I, pp. 109-133. Editorial Planeta, Buenos Aires.

Sacur Silvestre, R., N. Buc, A. Acosta y D. Loponte 2013. Estrategias de captura de presas y sistemas de armas de los cazadores-recolectores que habitaron el humedal del Paraná inferior: Una aproximación experimental y arqueológica. Comechingonia 17:27-57.

Schmidl, U. 1948. Crónica del Viaje a las Regiones del Plata, Paraguay y Brasil, Schmitt, D.N. y K.D. Lupo, Buenos Aires.

Serrano, A. 1972. Líneas Fundamentales de la Arqueología del Litoral (Una Tentativa de Periodización). Instituto de Antropología, Córdoba.

Sneath, P. H. y R. Sokal 1973. Numerical Taxonomy. Freeman, San Francisco.

Stark, M. 1998. The Archaeology of Social Boundaries. Smithsonian Institution Scholarly Press, Washington, D.C.

Torres, L.M. 1903. El Cementerio Indígena de Mazaruca (Entre Ríos). Cabaut, Buenos Aires.

Torres, L.M.1911. Los Primitivos Habitantes del Delta del Paraná. Universidad Nacional de La Plata, Biblioteca Centenaria, Buenos Aires. 
Vignati, M.A. 1941. Censo óseo de paquetes funerarios de origen Guaraní. Revista del Museo de La Plata. Antropología 9(2):1-19.
Zimmerman, G., H. Goetz y P. Mielke JR. 1985. Use of an improved statistical method for group comparisons to study effects of prairie fire. Ecology 66:606-611.

\section{Nota}

1 No se cuenta con datos publicados sobre este sitio. Por lo tanto, la referencia corresponde a la fecha de ingreso de las colecciones de dicho sitio al Museo Etnográfico. 


wWw.androsimpresores.cl 
